\title{
Robert BOILY
}

professeur au département de science politique, Université de Montréal

(1988)

\section{"La transformation du Parti libéral du Québec sous Georges-Émile Lapalme (1950-1958)"}

Un document produit en version numérique par Jean-Marie Tremblay, bénévole, professeur de sociologie au Cégep de Chicoutimi

Courriel:jmt_sociologue@videotron.ca

Site web: http://www.uqac.ca/jmt-sociologue/

Dans le cadre de la collection: "Les classiques des sciences sociales"

Site web: http://www.uqac.ca/Classiques_des_sciences_sociales/

Une collection développée en collaboration avec la Bibliothèque Paul-Émile-Boulet de l'Université du Québec à Chicoutimi Site web: http://bibliotheque.uqac.ca/ 
Cette édition électronique a été réalisée par Jean-Marie Tremblay, bénévole, professeur de sociologie au Cégep de Chicoutimi à partir de l'article de :

\section{Robert Boily,}

[professeur au département de science politique, Université de Montréal.]

"La transformation du Parti libéral du Québec sous Georges-Émile Lapalme (1950-1958)".

Un article publié dans l'ouvrage publié sous la direction M. Jean-François Léonard, Georges-Émile Lapalme, pp. 221-237. Montréal: PUQ, 1988, 303 pp. Montréal: Les Presses de l'Université du Québec, 1988, 303 pp. Collection: Les leaders politiques du Québec contemporain.

[Autorisation de l'auteur accordée le 22 juin 2005.]

쇼 Courriel : robertboily@,videotron.ca

Polices de caractères utilisée :

Pour le texte: Times, 12 points.

Pour les citations : Times 10 points.

Pour les notes de bas de page : Times, 10 points.

Édition électronique réalisée avec le traitement de textes Microsoft Word 2001 pour Macintosh.

Mise en page sur papier format : LETTRE (US letter), 8.5' $\mathrm{x} 11$ '”)

Édition complétée le 20 juillet 2005 à Chicoutimi, Ville de Saguenay, province de Québec.

\section{Fait avec}




\section{Robert Boily}

[professeur au département de science politique, Université de Montréal.]

\section{"La transformation du Parti libéral du Québec sous Georges-Émile Lapalme (1950-1958)"}

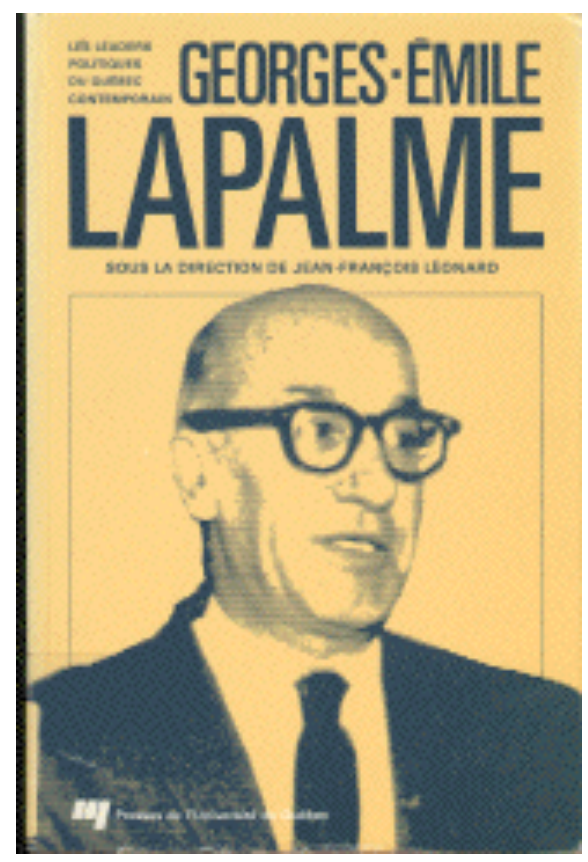

Un article publié dans l'ouvrage publié sous la direction M. Jean-François Léonard, Georges-Émile Lapalme, pp. 221-237. Montréal: PUQ, 1988, 303 pp. Montréal: Les Presses de l'Université du Québec, 1988, 303 pp. Collection: Les leaders politiques du Québec contemporain. [Autorisation de l'auteur accordée le 22 juin 2005.] 


\section{Table des matières}

Introduction

Une période de transition

1950-1958 : Un projet inachevé mais déjà déterminant

$\underline{\text { Le parent pauvre de la famille libérale }}$

Le modèle projeté : un nouveau type de parti

Une transformation inachevée. Un mouvement irréversible

Ampleur de la résistance : le deuxième front

La fédération c'est le parti?

Nous le parti, vous la fédération

Indépendance ou souveraineté-association

Le Parti Libéral fédéral et Duplessis

Les comportements ambigus du parti libéral provincial et de la fédération

Comment assurer l'autonomie dans l'association?

$\underline{\text { Références }}$ 


\section{Introduction 1}

$\underline{\text { Retour à la table des matières }}$

"Sans se perdre dans le dédale des analyses qui seraient tout de même incapables de nous offrir une réincarnation de ce qui a fui, les fouilles du chercheur, qu'il soit sociologue ou politicologue, dans les textes officiels reconstituent à la manière des ouvrages intitulés : "La vie quotidienne au temps de..." le climat d'une petite civilisation perdue dans une grande". (Le Vent de l'oubli. Mémoires. II, p. 72).

Tout n'a pas commencé le 20 mai 1950, au moment du choix de GeorgesÉmile Lapalme comme chef du Parti libéral provincial. Depuis l'Action libérale nationale et l'expérience du Bloc populaire, au sein même du Parti libéral, une volonté de transformation s'est exprimée, a réclamé une démocratisation du parti. $\mathrm{Au}$ cours de la brève période qui suivit, soit jusqu'au moment du départ de Georges-Émile Lapalme et de l'élection de Jean Lesage à la tête du parti le 31 mai 1958, l'appel à la nécessité et à l'urgence d'une démocratisation de la politique au Québec et notamment de la vie partisane, s'exprimait également à l'extérieur du Parti libéral, au sein des syndicats, d'un journal comme Le Devoir, chez des intellectuels comme Isocrate, observateur sagace de cette période, dans des groupes de gauche comme le Parti social démocrate ou des mouvements politiques comme le Rassemblement. Malgré le scepticisme profond de ces divers observateurs concernant la volonté réformiste du Parti libéral et leurs critiques sévères touchant la lenteur de cette transformation, on ne peut sous-estimer le

Nous tenons à remercier tout particulièrement $\mathrm{M}$. Henri Dutil pour l'interview éclairante qu'il nous a accordée et pour nous avoir permis d'utiliser ses archives. C'est cette documentation qui, pour une large part, est à la base même de ce texte. Nous remercions également $\mathrm{M}$. Yoland Sénécal pour sa collaboration dans le dépouillement des journaux. 
caractère déterminant pour le Parti libéral du Québec et pour le système partisan québécois, de l'action entreprise, dans des conditions éminemment difficiles, par un petit groupe de réformistes.

Rejetant l'option d'un nouveau parti, ils ont fait le choix d'un changement par l'intérieur. Ils vont donner ainsi naissance à un nouveau type de parti qui ne connaîtra son parachèvement qu'au début des années 70, sous la forme d'un parti de rassemblement et sous le nom de Parti libéral du Québec. D'autre part, le réformisme social, économique et politique qui anima ce parti et donnera naissance à la "Révolution tranquille", a provoqué également une modification fondamentale du système de partis: l'introduction d'une distance idéologique entre les projets de société défendus par les deux principaux partis en présence, une dimension absente depuis la fin du XIXe siècle.

\section{Une période de transition}

$\underline{\text { Retour à la table des matières }}$

Entre le Parti libéral dont hérite Georges-Émile Lapalme et celui dont Jean Lesage prend la direction quelque huit années plus tard, la différence est énorme, dans les structures, l'esprit, les orientations. Entre le modèle que l'on peut, avec prudence, dégager des intentions exprimées et la réalité, entre le dire et le faire, une distance existe également tout au cours de la période. En 1958, la transformation n'est pas complétée. Le mouvement est devenu cependant irréversible: les objectifs poursuivis ne seront certes pas tous atteints, mais le parti toutefois ne pourra plus être ce qu'il a été.

Cet écart entre le modèle projeté et la réalité tient essentiellement à deux groupes de facteurs : le premier correspond aux nombreux points de résistance au changement; le deuxième, aux nombreuses ambiguïtés concernant plusieurs aspects fondamentaux de la réforme souhaitée. C'est que le modèle que nous venons d'évoquer n'a pas eu, dans la réalité, toute la cohérence du construit analytique que nous tirons du vécu observé. Ce modèle réfère à toute une série d'objectifs exprimés, de geste parfois posés mais non exempts de contradictions. Par touches successives et sans que les réformistes partagent les mêmes points de vue sur les diverses questions, la transformation du Parti libéral a été poursuivie. C'est l'addition de tous ces gestes aux effets parfois insoupçonnés, c'est la décantation des objectifs exprimés et du vécu qui donnent à l'ensemble une signification. Il faut donc prendre garde de ne pas forcer le réel pour y lire le profil d'un développement rationnel et unidimensionnel. De fait, la réalité n'a pas 
eu cette clarté. Le changement fut plus complexe, comme c'est toujours le fait d'une période de transition, c'est-à-dire une période pendant laquelle le passage d'un type de situation à un autre s'opère, provoquant ainsi la coexistence à des degrés variables des caractères anciens et des nouveaux. Par définition, dirionsnous, une telle période ne peut avoir la cohérence que possédait le modèle ancien qui résiste et que possédera le modèle nouveau qui progressivement s'implante. Généralement, de telles périodes sont animées par des personnes qui appartiennent aux deux mondes en fusion. Ce qui est particulièrement le cas de Georges-Émile Lapalme.

À vrai dire cette période de transition pour le Parti libéral n'est pas tout à fait terminée au moment du départ de Georges-Émile Lapalme ; elle aura traversé, cependant, sa phase la plus significative, même si on peut considérer que ce n'est que par l'intégration en 1971 de la Fédération et des autres organes du parti en une seule structure, le Parti libéral du Québec, que le processus se terminera, officialisant ainsi la transformation opérée, soit le passage d'un parti de notables à un parti de rassemblement.

\section{0-1958 : \\ Un projet inachevé mais déjà déterminant}

\section{Le parent pauvre de la famille libérale}

\section{$\underline{\text { Retour à la table des matières }}$}

A son arrivée à la tête du Parti libéral provincial c'est un parti à la fois inchangé et différent que Georges-Émile Lapalme trouve. Parti inchangé, dans la mesure où le profil traditionnel de ce parti de notables qui était, depuis le début, celui du Parti libéral continuait de prévaloir : toute-puissance du chef et des grands organisateurs, financement occulte, aile parlementaire constituant le seul élément visible et quelque peu permanent, relations électeurs, militants et parti relevant du clientélisme. Au fond, un parti non seulement peu différent de celui de Taschereau, mais aussi de celui de l'Union Nationale.

Pourtant, sous d'autres aspects, le parti dont hérite Georges-Émile Lapalme est fort différent, à la fois sur le plan interne (organisation, moyens, assise électorale) et sur le plan externe (relations avec le Parti libéral national). 
Depuis la défaite de 1936, et mise à part la parenthèse Godbout de 1939-1944, le Parti libéral est en chute libre, accumulant les défaites et n'arrivant pas à sortir du discrédit de la fin de l'ère Taschereau. L'aile parlementaire c'est, en 1950, huit députés, avec comme point d'appui $38 \%$ de l'électorat. La caisse électorale est vide, l'organisation en déroute dans la majorité des circonscriptions, et les permanences de Québec et de Montréal dans un état complet de dénuement complet (un taudis écrira Lapalme au sujet des locaux de Québec). À Montréal, les libéraux fédéraux leur fournissent, non sans mal, un pied-à-terre.

À l'intérieur de l'étroite symbiose des ailes provinciale et fédérale du Parti libéral au Québec, notamment au point de vue l'organisation, malgré la fluidité des frontières, Taschereau avait su, parallèlement à la défense de l'autonomie du Québec, assurer la primauté des libéraux provinciaux. À la tête d'un parti au pouvoir depuis 1897 et face à un Parti libéral fédéral moins stable dans ses assises, Taschereau avait été à même de fournir les hommes et l'argent au moment des élections fédérales en plus d'assurer sa pleine autonomie au moment des élections provinciales.

C'est à une situation complètement inversée que doit faire face Georges-Émile Lapalme. Son parti est dans l'opposition, démuni et discrédité. Au fédéral, le Parti libéral règne en maître incontesté de 1935 à 1957 sous la direction de Mackenzie King, puis de Louis Saint-Laurent. Le Parti libéral provincial est devenu le parent pauvre. Comme au temps de Laurier, le chef du Parti libéral est à Ottawa ; c'est vers Ottawa que se tournent les jeunes libéraux qui veulent faire carrière, comme Lapalme et Lesage le feront. Cette nouvelle situation entraîne pour le Parti libéral provincial une perte de leadership au sein du monde libéral québécois et, à tout le moins, un risque accru de dépendance quant à ses moyens d'action.

\section{Le modèle projeté : un nouveau type de parti}

$\underline{\text { Retour à la table des matières }}$

Plus qu'à travers les mémoires, qui parfois, avec la distance, offrent une vue simplifiée des choses, c'est à travers les discours du temps, les procès-verbaux des divers organismes de la Fédération, notamment, que nous pouvons nous faire une idée de ce rêve en voie de réalisation. Un certain nombre de thèmes reviennent, avec des variantes, qui nous permettent de dessiner les grands traits de ce projet. Il faut toutefois être conscient que par ce précédé nous risquons de donner au projet une apparence de trop grande cohérence. Nous corrigerons par la suite. En effet, 
ce modèle que nous tentons de reconstruire, réfère aux dires plutôt qu'au faire, aux objectifs réduits à leur dénominateur commun, plutôt qu'à leur réalisation.

C'est à partir de l'objectif général de la démocratisation que la transformation est poursuivie. Il faut, pour abattre la dictature duplessiste et pour garantir qu'un retour au pouvoir des libéraux ne répète l'histoire, construire un instrument adapté à de tels objectifs, c'est-à-dire un parti nouveau. Il faut faire en sorte que le chef du parti ne soit plus imposé, par un establishment ni fédéral ni provincial (organisateurs, trésoriers, sénateurs, etc.). Les congrès du parti, lorsqu'ils ont lieu, doivent échapper aux détenteurs traditionnels du pouvoir qui contrôlent des délégués qu'ils ont souvent choisis et dont ils ont payé les dépenses. Démocratiser, c'est aussi assainir le financement des partis. Il faut que le Parti libéral provincial et les partis en général échappent aux puissances de l'argent. Comment penser que, de retour au pouvoir, on puisse avoir autrement les mains libres ? C'est là un des thèmes majeurs défendus par J.-M. Nadeau. Il faut que ce parti soit autre chose qu'une machine électorale. Il lui faut s'alimenter au savoir et développer une pensée politique qui progressivement articule les diverses facettes du Québec à construire. A ce processus de réflexion et de diffusion de choix politiques, il faut associer les militants libéraux. Il faut donc opérer dans le parti un changement de pouvoir, de mentalité; il faut convertir les travailleurs d'élection en militants, sans perdre de vue toutefois que la bataille électorale demeure le passage obligé vers la victoire.

Il faut donc créer un organisme qui ait une permanence dans ses structures, qui permette une continuité dans l'action; un organisme qui, dans ces mêmes structures, colle le mieux possible aux nécessités de l'action électorale. D'où cette fameuse pyramide dont Lapalme et les autres évoquèrent progressivement et de plus en plus clairement la construction. La base : les bureaux de scrutin regroupés en associations de municipalités ou de quartiers et de là en associations de comtés, puis en associations régionales. C'est le regroupement de ces diverses associations régionales qui forme la Fédération libérale provinciale du Québec. Les dirigeants de la Fédération, comme ceux des autres niveaux, doivent avoir dans leur élection par les délégués en congrès une base démocratique. Forcés de rendre compte en congrès de leur mandat, ils deviennent responsables. La participation des militants, à travers leurs associations locales, régionales, le congrès annuel ou les comités permanents, associe l'ensemble du parti à l'élaboration des orientations politiques. La carte de membre officialise l'adhésion, le paiement d'une cotisation de deux dollars amorce la démocratisation du financement. Enfin, soulignons que cette volonté s'exprime également dans les propositions de réforme des lois électorales et dans celle de la fonction publique afin de modifier les relations existant jusque-là entre le parti au pouvoir et l'État. Dernier élément, mais non le moindre, il faut assurer au parti provincial sa pleine autonomie face au Parti libéral fédéral. 
La structure proposée et mise en exécution à partir de la fondation de la Fédération en 1955, tentait donc d'allier les objectifs démocratiques à ceux d'une plus grande autonomie du parti provincial et aux nécessités d'une lutte électorale. La base de la structure n'est pas sans rappeler celle des partis américains, alors que le niveau intermédiaire et le sommet, de même que l'esprit général, semblent emprunter davantage aux partis socio-démocrates d'origine européenne.

Il y avait dans cette volonté de transformation fondamentale du parti, l'espoir que sa démocratisation le rendrait crédible auprès de l'électorat en général, mais aussi auprès des nombreux groupes d'opposition au duplessisme : intellectuels, syndicats, groupes de gauche, etc. qui continuaient à douter de cette démocratisation. Le Parti libéral affrontait le scepticisme conjoint des nationalistes qui doutaient de sa volonté autonomiste face au fédéral et des groupes de gauche qui mettaient en doute son progressisme et sa démocratisation. Il répondait peu encore à ce moment, du moins de manière explicite, à ceux qui allaient devenir par la suite son élément moteur, son appui le plus dynamique et le plus créateur, soit ceux qui tentaient l'intégration du nationalisme et du progressisme.

\section{Une transformation inachevée. Un mouvement irréversible}

Au moment où Jean Lesage succède à Georges-Émile Lapalme et entreprend les dernières étapes vers la victoire de 1960, le processus de transformation n'est pas terminé. Entre le modèle projeté, réaffirmé régulièrement, et la réalité des écarts subsistent. Par contre, entre le Parti libéral de 1950 et celui de 1958 quel chemin parcouru ! Une structure existe à l'échelle du Québec, aux niveaux local, régional et provincial. Elle a été développée difficilement, progressivement, mais les résultats sont là : si, en 1955, après la formation de la Fédération, il n'y avait que 10 comtés organisés en association dans le district de Québec et guère plus dans celui de Montréal, en 1957 on estime à 72 le nombre total de comtés où existe une association affiliée à la Fédération et en 1958 il ne reste que 14 des 93 comtés de la province à organiser. Il s'agit surtout de comtés urbains. Des statuts existent qui définissent les pouvoirs et les fonctions de chaque composante, des statuts qui servent d'assise à des contestations de décisions ou de comportements. Des congrès annuels rythment la vie du parti, donnant aux militants la possibilité de s'exprimer. Il est vrai cependant que le poids des commissions demeure très 
lourd, notamment celui de la commission politique ; que les militants ont peu de temps pour étudier et discuter les résolutions qui leur sont présentées par la commission responsable. Il n'en reste pas moins qu'ils ont l'occasion de protester, de revendiquer, de proposer et que des procès-verbaux gardent trace de tout cela.

En haut de la pyramide, on parle "institut de recherche", journal, comité de formation, mise sur pied de cellules de réflexion politique sur l'ensemble du territoire, pouvoir du militant. A la base, cependant, malgré une grande générosité au moment des congrès, l'action politique quotidienne tarde à venir, l'engagement semble demeurer difficile. Le financement, même s'il s'est diversifié, n'est pas vraiment démocratique ni dans ses sources ni dans son contrôle; l'autonomie pleine et entière n'aura lieu qu'au congrès de 1964 et c'est lors d'un congrès, qui pour une part échappe à la Fédération que le nouveau chef du parti sera élu en 1958.

Ces contrastes de la réalité partisane libérale, ces décalages entre les affirmations et les réalisations sont le propre d'une période de transition. Ils s'expliquent tout autant par la résistance au changement que par l'ambiguïté que nous pouvons déceler touchant plusieurs des objectifs avancés par ceux qui recherchent ce changement. Dans la plupart des cas, ces deux groupes de facteurs sont étroitement entremêlés, même si pour fin d'analyse nous tentons ici de les séparer ; c'est cette réalité qui donne à la dynamique de cette phase de transition son caractère propre.

Après avoir illustré l'importance de cette résistance au changement, nous nous attacherons à deux questions qui tout au cours de cette période, comme après d'ailleurs, ont animé fortement les débats: la Fédération, est-ce le parti ? Indépendance ou souveraineté-association du Parti libéral provincial face au Parti libéral fédéral ?

\section{Ampleur de la résistance : le deuxième front}

$\underline{\text { Retour à la table des matières }}$

La réalité et l'ampleur de la résistance à la transformation du Parti libéral ne font aucun doute, de même que les tensions et le manque d'unité que cette résolution a entraînés. Les observateurs de l'époque l'ont fortement souligné et dénoncé plus d'une fois. Il faudrait rappeler ici les commentaires sévères d'un "Isocrate", d'un Laurendeau, d'un Filion ou d'un Pierre Laporte à l'occasion de 
chacun des congrès de la Fédération. Au sein même du parti, on dénonce ce deuxième front qu'entretiennent les libéraux soit provinciaux soit fédéraux, alors que la lutte est déjà si difficile contre Duplessis et son régime. À chacun des congrès, on lance un appel à l'unité. Nulle part, ailleurs, peut-être que dans son discours d'adieu, Georges-Émile Lapalme comme chef du parti, n'a exprimé avec autant de brutale franchise et d'amertume l'impact de cette guerre interne du Parti libéral.

Cette résistance au changement n'a pas été le seul fait de l'establishment libéral, qu'il soit fédéral ou provincial ; tous les fédéraux n'étaient pas contre la Fédération. Ce fut également le fait d'un électorat et de militants peu habitués à autre chose qu'à lutter pour le pouvoir, quels qu'en fussent le prix et les moyens utilisés. On ne modifie pas si facilement des habitudes, des comportements, des styles ancrés dans une pratique déjà fort ancienne. La tâche est encore plus ardue lorsque les recettes nouvelles proposées ne donnent pas de résultats immédiats et que, chez l'adversaire, tous les moyens sont bons pour vaincre. Comment croire en l'urgence d'un parti différent de l'U.N. si seules les armes de l'adversaire permettent d'obtenir le pouvoir?

L'importance d'un programme politique de changement, la nécessité d'une vaste organisation à caractère permanent, en un mot tout ce qui correspond au nouveau projet libéral, au message que tente de faire passer G.-É. Lapalme dans ses tournées des régions du Québec, toutes ces idées passent difficilement. Ce que nombre d'auditeurs préfèrent, c'est l'emballage, c'est-à-dire l'attaque virulente contre l'ennemi, l'U.N. Dans ses mémoires, G.-É. Lapalme se demandera, des années plus tard: "mais, au sein des militants, ai-je jamais fait disparaître la notion de pouvoir pour le pouvoir" (Le Vent de l'oubli. Mémoires. II, p. 51).

Autre manifestation de cette difficulté de changer les mentalités, l'échec du financement populaire. En effet, comme pour le Bloc populaire, les campagnes de souscription populaire n'ont pas donné de résultats positifs. Les militants ne semblaient pas comprendre la signification d'un tel mode de financement. lis n'ont pas davantage compris la signification du paiement d'une cotisation obligatoire de deux dollars pour une carte de membre officiel de la Fédération. Si on acceptait l'idée d'une organisation permanente, d'un membership visible et contrôlé, accordant des droits au sein du parti, on comprenait mal qu'il faille payer pour cela. De fait, tout au cours de cette période de transition, il ne fut jamais vraiment possible d'imposer cette cotisation comme le prévoyait la constitution de la Fédération. La tolérance et la souplesse furent de mise.

Autre forme de résistance, celle des souscripteurs traditionnels. Dans leur réalisme et conformément à leur culture politique, ils donnaient à celui qui avait le plus de chance de rapporter des dividendes, c'est-à-dire au Parti libéral fédéral et à l'Union Nationale. Ils hésitaient à fournir leur quote-part traditionnelle à l'autre parti qui formait l'Opposition. De plus, les politiques préconisées sur les plans 
social et économique inquiétaient. Incapable finalement de se financer complètement et encore plus d'assumer le coût d'une élection, la Fédération devra donc faire appel plus d'une fois à la caisse électorale du parti fédéral, celle dont elle veut précisément s'affranchir.

Dernier exemple de ces foyers de résistance qui témoignent de la difficulté de changer les mentalités : le choix des candidats. Les réformistes tenaient à ce que les candidats soient choisis démocratiquement par des conventions; ils cherchaient aussi des candidats conformes à leurs objectifs, capables de lutter pour ces idéaux. Si l'on en croit le témoignage de G.-É. Lapalme, il fut extrêmement difficile de négocier les candidatures avec des militants locaux qui préféraient récompenser quelque organisateur dévoué :

Par des informations et des regroupements divers, nous nous dirigions vers des compétences qui parfois acceptaient, si elles étaient choisies par une convention locale, de porter le drapeau. Commençaient alors, avec les dirigeants du comté, les palabres désespérantes. "Oui, monsieur X est le meilleur homme, mais les libéraux n'en voudront pas. Il n'est pas un gagnant. C'est un tel qui devrait être candidat." Et nous nous trouvions devant un gros Jean de la politique de clocher, ignare ou à peine dégrossi, mettant de l'avant avec orgueil ses 25 ans de libéralisme actif et sa mainmise totale sur l'organisation du lieu. Les vieux militants, partisans de l'immortalité, rompus aux vieux trucs de la guerre des tribus, gardant pour eux seuls les secrets du parti, invoquaient leur passé pour pouvoir du haut d'un siège de député participer à la curée et reprendre le temps perdu. (Le Vent de l'oubli, Mémoires, II, p. 109).

Le Parti libéral provincial, ne réussissant pas à rétablir sa crédibilité auprès des divers groupes d'opposition, malgré tous ses efforts et ses appels, arrivera tout aussi difficilement à attirer vers lui des forces nouvelles, du sang nouveau. Cette fois ce n'est pas une résistance au changement, c'est plutôt la résistance à un parti qui à leurs yeux n'a pas suffisamment changé. Il faudra du temps pour que l'ouverture se fasse.

Donc qu'il s'agisse de l'importance d'un programme pour la lutte politique, de la nécessité d'une nouvelle organisation et d'un mode de financement populaire ou encore du choix des candidats, des réticences tenaces, pour ne pas dire des résistances très fermes, se sont manifestées au sommet comme à la base du parti. L'étude de deux dimensions particulières permet de mieux saisir la complexité de ce processus de changement. 


\section{La fédération c'est le parti ?}

$\underline{\text { Retour à la table des matières }}$

La Fédération, est-ce le parti ? Force est de répondre non, même si M. Lapalme a affirmé le contraire à de nombreuses reprises, de même que d'autres dirigeants de la Fédération. C'est à côté des structures existantes que la Fédération s'est développée, et au lendemain du départ de M. Lapalme on s'interrogeait, tout autant qu'au lendemain de la fondation de la Fédération, sur les limites du pouvoir de la Fédération au sein du Parti, sur la spécialisation des tâches de l'organisation (structure à fonction électorale : financement, propagande, comité juridique, etc.) et de celles de la Fédération. Or, une transformation complète du Parti libéral provincial dans la perspective d'une démocratisation n'appelait-elle pas une substitution des nouvelles structures aux anciennes ? La persistance des anciennes structures s'explique-t-elle uniquement par les résistances au changement?

Nous avons vu quelle était la situation générale du Parti libéral provincial au moment de l'arrivée de G.-É. Lapalme. Avant la naissance de la Fédération, il y avait ici et là à travers le Québec des regroupements de libéraux, associations locales ou régionales, bona fide, sans membership officiel et sans permanence. Existaient également un regroupement des femmes libérales et un regroupement des jeunes libéraux, notamment dans les universités. Il y avait enfin les organisations des districts de Montréal et de Québec, chargées du financement et de la conduite des luttes électorales fédérales et provinciales, avec l'appui des organisateurs régionaux et locaux. Au sommet, le chef, maître des décisions, des nominations aux postes principaux du parti ; à ses côtés, l'aile parlementaire. Le Parti libéral d'avant la Fédération, c'est cette nébuleuse, aux frontières relativement floues mais à la structure de pouvoir pourtant réelle.

C'est à l'intérieur de ce parti, mais à côté des structures existantes, que la Fédération déploiera difficilement sa propre structure, sans englober les fédérations de femmes ou de jeunes, ni les organisations des districts de Montréal et de Québec qui dépendaient directement du chef.

Au congrès de 1957 , les délégués se retrouvèrent devant un organigramme du parti préparé à la demande de M. Lapalme par G. Brady, chef du secrétariat de la Fédération. De cet organigramme et du rapport qui l'accompagne, la coexistence sous l'autorité du chef des structures d'organisation et de celles de la Fédération 
ressort clairement. Dans son rapport, G. Brady fait remarquer qu'il y a, sur plus d'un point, superposition, dédoublement des rôles et que personne n'a jamais jusque-là établi clairement les fonctions de chaque commission ou de chaque comité. Il lui apparaît donc nécessaire, dans la mesure où l'on entend maintenir cette double structure, de mettre sur pied, auprès du chef, un comité directeur voué à la coordination des deux grandes structures.

Les décisions du congrès sont représentatives de ce que nous appelons cette phase de transition. D'une part, on supprima dans l'organigramme la référence au trésorier pour ne parler que d'un comité des finances, on créa des postes de viceprésidents pour une représentation des femmes, des jeunes et des autres groupes ethniques, ce qui va dans le sens d'une meilleure intégration de l'ensemble des organismes du parti ; d'autre part, on supprima la proposition de mise sur pied d'un comité directeur et on donna son accord à l'organigramme ainsi modifié. La coexistence des structures est ainsi officialisée, sans que soient résolus les problèmes du dédoublement des fonctions et de la persistance d'organismes tels que la Fédération des femmes ou celle des jeunes. Il est vrai qu'on a reconnu à ces deux fédérations une représentation de droit au sein des organismes de direction de la Fédération provinciale et au sein des délégations de comtés lors des congrès.

La création de la Fédération, elle-même une structure complexe, a donc rendu encore plus complexe la structure générale du Parti libéral, plus difficile pour le chef du parti I'harmonisation des actions de l'ensemble des corps constituants, corps sur lesquels il n'avait en vérité qu'une autorité très relative. Le rôle de la Fédération libérale des jeunes dans le départ de M. Lapalme illustre bien ce fait.

Ainsi, par suite de l'action soutenue des opposants à la Fédération ou à son expansion sur la totalité du territoire, par suite de la résistance passive des militants libéraux et des électeurs en général à un changement de mentalités et de comportements, la Fédération a certes connu des difficultés dans la mise en place de sa propre structure, toutefois elle est demeurée une composante, importante de l'ensemble du Parti libéral provincial.

La Fédération n'était pas le parti. De fait, elle ne le deviendra jamais. Si la Fédération n'a pas réussi à se substituer au Parti, ce n'est pas uniquement à cause des résistances au changement, mais également parce que ses promoteurs n'ont pas fait preuve d'une volonté aussi affirmée, d'une conception aussi radicale des choses. De nombreuses manifestations en témoignent, tout au cours de la période. Ainsi, observe-t-on aux niveaux local et régional, un pouvoir réel de résistance à l'expansion de la Fédération. 


\section{Nous le parti, vous la fédération}

$\underline{\text { Retour à la table des matières }}$

L'idée de la Fédération est née au congrès de 1950, aime-t-on constamment à rappeler au cours de ces années de développement de la Fédération. En fait, ce qui ressort du congrès de 1950, c'est l'idée d'une association libérale provinciale, répondant entre autres aux objectifs principaux de deux candidats à la direction, MM. Nadeau et Philippon. Cette décision, c'est le résultat d'un compromis mis de l'avant par George Marler afin d'écarter le projet d'un conseil supérieur du Parti qui aurait eu autorité sur le chef. On veut donc une association, mais il ne faut pas toucher aux pouvoirs du chef. Faut-il s'étonner que, par la suite, après la naissance de la Fédération, aussi bien Georges-Émile Lapalme que Jean Lesage, chefs du parti, disent nous en parlant du Parti et vous en parlant de la Fédération ? Ce statut particulier du chef, comme extérieur à la Fédération, au-dessus d'elle, est un facteur qui explique le maintien du parallélisme des structures puisque c'est le chef qui désigne les détenteurs des postes au sein de la structure d'organisation et que ces dernières ne sont vraiment responsables qu'à lui. Dans un cas, celui de la Fédération, les détenteurs de postes de direction correspondent à une conception démocratique du pouvoir. Ceux de l'organisation continuent de répondre à une conception traditionnelle de caractère autoritaire. À l'intérieur d'un même parti, on a donc créé la confrontation de deux conceptions fondamentales du pouvoir, la confrontation de deux légitimités.

Les discussions qui animent les premiers exécutifs au lendemain du congrès de formation de la Fédération, où avait été fortement affirmée sa vocation à devenir le parti tout entier, montrent clairement que tout est loin d'être aussi simple, aussi limpide. Pour certains, cette vocation ne fait pas de doute; pour d'autres, des interrogations demeurent. Les organisations des districts de Montréal et Québec sont-elles extérieures à la Fédération ? Si non, elles relèvent de la Fédération, leurs organisateurs en chef devraient siéger à l'exécutif. Le chef du secrétariat de la Fédération, qui a ses bureaux à l'organisation du district de Montréal, doit-il porter le titre d'adjoint à l'organisateur ou un autre titre ? La Commission politique, dont le rôle sera toujours déterminant au sein du Parti libéral, et dont le responsable avait toujours été jusque-là nommé par le chef, 
estelle un organisme extérieur à la Fédération, indépendant de la Fédération ou soumis à l'autorité de cette dernière ?

Progressivement, les divers comités et commissions de la Fédération seront sans ambiguïté reliés à la Fédération, notamment sous l'autorité de l'Exécutif et du Conseil général. Une articulation plus étroite se développera entre la Fédération des femmes, celle des jeunes et la Fédération libérale provinciale. Pourtant, les nombreux rappels à l'ordre pendant cette période montrent que cette intégration à l'intérieur même de la structure de la Fédération n'a pas été facile. Par ailleurs, tout ce qui était relié à l'organisation demeura à l'extérieur de la Fédération. L'aile parlementaire elle-même. demeura largement extérieure à la Fédération, bien que quelques rencontres spéciales furent organisées entre elle et les organismes supérieurs de la Fédération. L'aile parlementaire relevait directement du chef. Son peu de pouvoir, à ce moment, dans la Fédération, témoigne moins de l'affirmation de la suprématie de la Fédération sur tout autre groupe, que de la coexistence de la Fédération avec les autres composantes du Parti.

Tout aussi significatif nous apparaît le processus suivi pour le remplacement de G.-É. Lapalme à la direction du Parti. Conformément à l'esprit nouveau, c'est à la direction de la Fédération que $M$. Lapalme remettra sa démission. La Fédération sera également associée à l'organisation du congrès spécial qui aura pour mission d'élire le nouveau chef. Ce qui peut étonner, c'est que l'on donnera également à ce congrès la mission de se prononcer sur le questions touchant l'orientation du Parti. Ce qui étonne davantage, c'est que M. Lapalme cherchera à justifier la convocation d'un congrès, dit plénier, au sein duquel voteraient tout un ensemble de délégués de droit (députés libéraux fédéraux, candidats libéraux fédéraux battus et sénateurs). La référence au congrès de 1950 peut, en effet, surprendre si on tient compte du fait qu'à cette époque aucune organisation libérale officielle et encore moins de structures démocratiques n'existaient. Comment justifier la tenue d'un tel congrès spécial lorsqu'une structure démocratique a été si péniblement mise sur pied ? Comment, paradoxalement, mieux que M. Lapalme exprimer l'idée que la Fédération n'est pas le parti ?

... Je viens annoncer aujourd'hui que j'ordonne la convocation d'un congrès plénier du Parti, ou, si vous aimez mieux, d'une convention du Parti libéral provincial de la province de Québec.

... Je dis qu'il faut un congrès plénier du Parti libéral provincial pour que tous les libéraux de la province de Québec dûment représentés dans chacun de leur comté puissent venir prendre part aux délibérations et essayer de régler les problèmes les plus cuisants.

D'autre part, j'ai été élu, moi, par une convention du parti. J'ai été élu par un congrès du parti, pas par une organisation, fût-elle aussi grande que la Fédération libérale provinciale. J'ai été élu par 1500 délégués venant de tous les comtés de la province de Québec et je dis qu'il appartiendra à tous les délégués de la province de Québec de régler tous les problèmes du Parti. Il n'est pas dans le pouvoir de la Fédération libérale provinciale de régler ces problèmes-là qui sont immédiats et qui sont pressants. Il 
appartient à tous les libéraux d'être représentés dans une convention plénière du parti, de faire là et alors parler la véritable voix du Parti libéral (Congrès de la Fédération libérale provinciale des 9 et 10 novembre 1957. Ouverture du congrès).

Il semble qu'il ait été difficile pour les délégués à qui on avait répété depuis déjà trois congrès que la Fédération c'était le parti, de comprendre ce qu'était cette "convention", ce congrès plénier, puisque M. Lapalme reviendra sur la question lors de son discours de clôture. Paradoxalement, à ce même congrès un attendu de la résolution qui proposait l'affiliation à la Fédération libérale nationale se lisait comme suit :

Attendu que la Fédération libérale de la province de Québec représente vraiment tous les libéraux de la province de Québec (Congrès de 1957. Rapport de la Commission de la constitution).

Au fond, pour plusieurs, la Fédération était un des instruments, un des organes du parti. Elle affirmait la permanence du parti entre les élections, permettait grâce à la participation des militants l'élaboration d'une pensée politique capable d'inspirer l'action politique du chef et plus tard du gouvernement, une fois au pouvoir. En retour, elle facilitait la diffusion de la pensée libérale auprès de l'électorat, tout en offrant une structure, notamment à la base, au niveau des municipalités et des bureaux de scrutin, prête à fonctionner au moment des élections. Plus globalement, la Fédération symbolisait la volonté à la fois de démocratisation et d'autonomie du Parti libéral provincial. Elle était la garantie morale du Parti.

D'autres organes du parti, liés plus directement à la lutte électorale, devaient mener à l'extérieur de la Fédération leur propre action. Il y avait donc une spécialisation des fonctions du parti qui tout naturellement reposait sur des structures différentes, mais sous l'autorité d'un seul chef. Les organisateurs en chef des districts électoraux de Montréal et Québec avaient la responsabilité de tout ce qui touchait la lutte électorale, cependant que les trésoriers voyaient à l'alimentation de la caisse électorale. La fonction programmatique appartenait davantage à la Fédération, ce qui n'était pas sans avoir un impact sur la fonction électorale de l'organisation. Quant à la fonction de relève, elle eut un caractère mixte, qu'il se fût agi du choix du chef ou de celui des candidats à la députation.

Une dernière illustration de cette ambiguïté qui a entouré la place véritable de la Fédération au sein du Parti libéral provincial pendant cette période, se situe au lendemain de l'accession de Jean Lesage à la direction du parti, alors que Georges-Émile Lapalme en est encore le leader parlementaire. Dès son accession à la direction, comme dans la période qui a suivi, Lesage a loué la Fédération, insisté sur la nécessité d'en achever l'implantation dans tous les comtés. Il s'est engagé à soumettre, à chaque congrès, son leadership à la confiance des militants. La Fédération c'est, dit-il, les états généraux du Parti libéral. C'est le discours. Au caucus convoqué par Jean Lesage et réunissant les membres de la députation et 
les dirigeants de la Fédération libérale provinciale le 10 juin 1958, toute l'ambiguiité de la situation ressort. La réunion a été précédée d'un caucus des députés. À nouveau le problème des fonctions, des rôles réciproques de la Fédération et des autres organes du parti est évoqué sans que des définitions claires puissent être fournies. Ce qui est tout aussi significatif, c'est que le nouveau chef affirme l'importance que doit avoir l'aile parlementaire dans le parti, notamment face à la Fédération, et qu'il cherche à mettre sur pied, prétextant leur caractère temporaire, des comités qui lui soient personnellement rattachés, même s'ils semblent dédoubler ceux de la Fédération. Mises à part les remarques de $\mathrm{M}$. André Rousseau, président de la Fédération ("À chaque congrès de la Fédération, il revient cette idée que la Fédération devrait être le parti dans tous les domaines"), les positions de M. Lesage ne paraissent pas avoir soulevé d'objections majeures.

Exprimant l'idée que les députés doivent être davantage associés à tous les rouages du parti, il ajouta :

C'est pour çà que j'ai tenu à avoir un caucus avec eux ce matin et un caucusconjoint, cet après-midi, afin que la Fédération prenne ses décisions suivant ce qui a été décidé ce matin.

Parlant du comité de finance - "qui est ma responsabilité" -qu'il veut mettre sur pied et qui par son membership régional apportera son support aux trésoriers, Jean Lesage précisa :

Je veux du sang nouveau. Je les connais personnellement. Je connais les capacités et l'honnêteté de ces gens-là. Je veux des gens dont je suis sûr ou dont mes amis sont sûrs". (Procès-verbal du caucus conjoint de la députation et des dirigeants de la Fédération, 10 juin 195 8).

Cette dualité de structures et de systèmes de légitimité ne pouvait demeurer encore longtemps telle quelle, car ses effets négatifs étaient nombreux : inconvénients du dédoublement des fonctions; conflits entre d'un côté la permanence de l'effort nécessaire à l'organisation électorale et au financement, et de l'autre, l'instabilité de la direction de la Fédération qu'entraîne le caractère, électif des postes ; coordination difficile par suite de la multiplicité des organes au sein du Parti, etc. Les réactions de Jean Lesage, dès son arrivée, sont de ce point de vue très significatives. La victoire de 1960, avant même que le processus de transformation ne soit complété dans les mentalités comme dans les structures, allait rapidement faire peser sur la Fédération un poids très lourd. C'est peut-être en pensant à cela que G.-É. Lapalme écrira :

Elle (la Fédération) n'est pas ce que j'aurais voulu qu'elle soit. Elle plie trop souvent sous le poids du dirigisme; c'est probablement la meilleure preuve qu'on la craint"... "Sa 
disparition serait une amputation mortelle pour le parti qui en a vécu. (Le Vent de l'oubli. Mémoires., Il, p. 192).

Il nous semble que ces diverses réalités ne permettaient pas une évolution vers un grand parti de masse comme le deviendra plus tard, pendant un temps, le Parti québécois. S'il pouvait difficilement demeurer dans les contradictions de la transition, le Parti libéral ne pouvait pas davantage revenir en arrière. Aussi c'est vers un type de parti qui permettait de concilier une participation des militants et une autorité centrale forte, une volonté- de proposer de grandes orientations politiques et une large implantation électorale assurant le pouvoir que ce parti évolua: maintien donc d'une fonction programmatique, mais soumise aux nécessités de la fonction électorale.

C'est donc à l'intérieur du modèle des partis de rassemblement que le processus de transition s'est achevé en 1971, alors que tous les organes ont été intégrés en une seule structure reposant officiellement sur une seule légitimité. Le Parti libéral du Québec représente, dans cette perspective, un des meilleurs exemples de la transformation d'un parti de notables en un parti de rassemblement.

\section{Indépendance ou souveraineté- association}

$\underline{\text { Retour à la table des matières }}$

Cette question de l'autonomie du Parti libéral provincial face à celui que l'on appela longtemps le Parti libéral national en est une des plus importantes et des plus débattues pendant cette période. Face à une Union Nationale qui s'est emparée du thème de l'autonomie provinciale et qui vante son indépendance du fait de son caractère essentiellement provincial, le Parti libéral provincial éprouvera de la difficulté à démontrer qu'il n'est pas ce que Duplessis appelait un parti fédéral-provincial.

Gérard Bergeron-" Isocrate" insistera sur la nécessité d'un parti à fonction provinciale. Le journal Le Devoir reviendra plus d'une fois sur cette nécessité. Au sein même du parti, le débat existe. C'est déjà un des thèmes majeurs de la course à la direction de 1950 et, tout au cours de la période, à l'occasion des tensions provoquées par les actions des libéraux fédéraux, le thème demeurera d'actualité. Il ne semble pas cependant que cette volonté d'autonomie soit allée jusqu'à une 
volonté d'indépendance totale à l'égard du Parti libéral fédéral ; une telle volonté fut certainement très minoritaire. On est plutôt devant de nombreuses expressions de degrés d'autonomie, qui visent toutes à permettre en même temps l'autonomie et l'association au parti national. On ne peut donc attribuer aux seuls comportements du Parti libéral fédéral la responsabilité totale du caractère très relatif de cette autonomie du Parti libéral provincial, même après la formation de la Fédération provinciale.

\section{Le Parti Libéral fédéral et Duplessis}

$\underline{\text { Retour à la table des matières }}$

Plus d'une fois le Parti libéral fédéral, au pouvoir à Ottawa, a, par ses agissements, placé le Parti libéral provincial dans de graves difficultés. Lorsque Louis S. Saint-Laurent donne raison à $\mathrm{M}$. Duplessis à propos des conditions d'exploitation du fer de l'Ungava, il place dans une situation fort difficile le Parti libéral provincial qui depuis des années s'oppose à ce type de développement des ressources naturelles; de plus, il s'agissait là d'une matière de juridiction provinciale. C'est à nouveau dans une position très inconfortable que fut mis le Parti libéral provincial lorsqu'après avoir appuyé Ottawa dans son refus de céder à Duplessis sur la question de l'impôt sur le revenu, il se retrouva devant l'entente Saint-Laurent-Duplessis, entente qui permettait d'éviter la double imposition et donnait la victoire à Duplessis.

Une question, cependant, souleva tout particulièrement la colère des libéraux provinciaux réformistes, et cela tout au long de la période retenue : ce fut celle dite des "collaborateurs". Dans un certain nombre de circonscriptions électorales fédérales, nombreuses aux dires de certains, les députés libéraux fédéraux étaient élus avec l'aide de membres de l'organisation de l'Union Nationale. Il y avait des pactes de non-agression. Au moment des élections provinciales, des candidats libéraux furent battus faute d'appui des organisateurs libéraux locaux. Les représentations faites par les libéraux de la province semblent n'avoir eu que peu d'effets sur leurs frères du Parti libéral national.

Bien sûr, ces divers agissements ont provoqué la colère des libéraux provinciaux. Pensons à la réponse de G.-É. Lapalme à Louis-S. Saint-Laurent : 
Il y a que l'Ungava est une chose essentiellement provinciale et que tous les accords que l'on a pu et que l'on pourra faire à ce sujet, sont du ressort exclusif de la province. Il appartient donc à la politique provinciale de les juger, de les discuter, de les approuver ou de les désapprouver et non pas à la politique fédérale dont les relations internationales ne sont pas toujours en conformité avec les besoins provinciaux. (Le Vent de l'oubli, Mémoires. Tome 11, 93).

Chacun de ces incidents a provoqué de vives tensions, incitant les réformistes à réaffirmer plus fort la nécessité d'assurer l'autonomie du parti. Il fallait aussi exclure du parti les collaborateurs, dira J.-L. Gagnon. Sarto Fournier, sénateur libéral élu à la mairie de Montréal avec l'appui de l'Union Nationale, connaîtra cette exclusion. Parallèlement à ce discours autonomiste, une série de gestes avaient cependant pour effet d'entretenir le flou des frontières entre les deux partis libéraux et de faciliter les attaques de Duplessis sur la dépendance des libéraux provinciaux.

\section{Les comportements ambigus du parti libéral provincial et de la fédération}

\section{$\underline{\text { Retour à la table des matières }}$}

Les deux chefs du Parti libéral provincial qui sont choisis au début et à la fin de la période retenue sont des députés fédéraux dont l'un, Jean Lesage, faisait partie, aux dires de certains, des collaborateurs. Dans le choix de G.-É. Lapalme comme candidat à la direction, les libéraux fédéraux ont joué un rôle fort important, et c'est un député fédéral, L.-R. Beaudoin, qui sera l'organisateur de sa campagne. Il demeurera organisateur pour le district de Montréal quelque temps après le congrès de 1950. Si Jean Lesage a joui de l'appui des fédéraux, leur rôle ne fut pas aussi déterminant que lors du choix de G.-É. Lapalme. Entre-temps, la Fédération avait été créée. Il n'en reste pas moins qu'aux deux congrès spéciaux qui ont élu Lapalme et Lesage, ont participé à titre de délégués de droit, les députés fédéraux, les candidats battus lors de l'élection fédérale précédente et les sénateurs libéraux. Nous n'insistons pas ici sur le rôle qu'ils ont pu jouer dans le choix des délégués; ce rôle semble avoir été plus déterminant en 1950 qu'en 1958, un très grand nombre de comtés dans ce dernier cas étant déjà formés en associations libérales rattachées à la Fédération libérale provinciale.

Lors des divers congrès de la Fédération, il y aura toujours parmi les invités d'honneur un représentant important du Parti libéral fédéral. Au congrès de fondation, ce sera Louis-S. Saint-Laurent. Au congrès de 1957, ce sera Lionel 
Chevrier qui parlera de l'unité nationale et de l'unité libérale. Lui succédant au pupitre des orateurs, G.-É. Lapalme ajoutera :

Et je suis très heureux qu'il ait prononcé ces paroles que vous avez entendues à la fin de son discours car il prononçait précisément, à ce moment-là, des paroles que j'aurais voulu prononcer moi-même. (Discours de clôture, Congrès de 1957, 10 nov.).

Une dernière illustration de l'ambiguïté entretenue par des gestes des libéraux provinciaux, concerne l'attitude de la Fédération au moment des élections fédérales. Ainsi, dans les mois précédant l'élection fédérale de 1957, la Fédération menait une campagne de financement. Afin d'avoir le champ libre, les libéraux fédéraux demanderont à la Fédération de suspendre sa campagne et négocieront une compensation financière en provenance du Parti libéral fédéral. De plus, moyennant une faible somme $(2000 \$)$, le chef du secrétariat de la Fédération travailla pour l'élection fédérale.

Ce type d'association faisait que, en plus de son action au niveau provincial, la Fédération consacrait une partie de ses efforts aux intérêts du parti fédéral. C'est ce que reconnaît J.-L. Gagnon, président de la Commission de la propagande et directeur de La Réforme, lorsqu'il souligne la tâche énorme qu'ont eu à abattre les dirigeants de la Fédération :

Il ne faut jamais oublier que la Fédération libérale provinciale a été fondée en novembre 1955, ici même à l'Hôtel Windsor. Depuis, nous avons eu, en juin 56, l'élection provinciale ; en novembre 56, le deuxième congrès de la Fédération; en juin 1957, l'élection fédérale; en novembre 57, le 3 e congrès de la Fédération; en janvier 58 , le congrès plénier du Parti libéral canadien; en mars 58, l'élection fédérale, en mai 58, le congrès plénier du parti provincial et, enfin, en novembre 58 , aujourd'hui, nous avons le 4e congrès de la Fédération.

Tout évidemment paralyse le travail de la Fédération en ce sens que, s'occupant des congrès, s'occupant d'élections, la Fédération ne pouvait pas tout faire, ne pouvait pas aller dans chaque comté porter ses messages, organiser des cadres là où c'était nécessaire et également tout en faisant une propagande politique, en s'occupant de la propagande des congrès, arriver toujours à faire une propagande sur le front de l'organisation. (Rapport de la Commission permanente de propagande et de publicité, Congrès des 1 et 2 novembre 1958).

Aussi ambiguës furent la proposition de faire de la Réforme un journal libéral fédéral au moment d'une élection fédérale et la décision de l'Exécutif de la Fédération, lors d'une réunion spéciale (4 octobre 1957), de permettre la participation des libéraux fédéraux au 3, congrès annuel de la Fédération libérale provinciale. 


\section{Comment assurer l'autonomie dans l'association?}

Retour à la table des matières

L'ambiguïté de ces comportements rendait certainement plus difficile chez les militants une perception claire de l'autonomie de la Fédération pourtant fortement exprimée au niveau du discours, et moins crédible chez les observateurs et les opposants au Duplessisme la distance effectivement prise avec le parti fédéral. La volonté de maintenir des liens étroits avec ce parti demeure l'orientation générale. $\mathrm{Au}$ fond, ce que le Parti libéral provincial tente pendant cette période, c'est de revenir à la situation antérieure, c'est-à-dire celle qui donnait la primauté à la structure provinciale dans la grande famille libérale du Québec et assurait ainsi son autonomie.

Ce qui se dégage des nombreuses discussions et interventions sur cette question, c'est l'idée d'une large famille libérale, englobant sans les confondre, le parti fédéral et le parti provincial, mais dont la base serait provinciale. C'est sur cette base que devaient s'élaborer les grands principes mais en assurant, au niveau de l'action, une spécialisation des tâches et des organisations. Une séparation complète au niveau de la direction confirmait cette autonomie.

Cette conception reflète donc une double volonté, celle de maintenir la collaboration au sein de la famille libérale, mais aussi celle d'empêcher le contrôle des fédéraux sur le parti provincial, On était particulièrement soucieux d'empêcher le phénomène des "collaborateurs". A cette fin, on a défendu le principe qu'il devait y avoir nécessairement une convention pour le choix d'un candidat à une élection fédérale et que cette convention devait reposer sur une organisation à base provinciale. Le procédé était le suivant : lors d'une élection fédérale, les associations provinciales officielles des divers comtés provinciaux englobés dans un comté fédéral, formaient l'association fédérale et choisissaient un comité responsable de la convention. On espérait, par là, empêcher la candidature de collaborateurs. De la même manière, afin d'assurer la primauté du parti provincial, on avait négocié avec la Fédération libérale nationale une procédure pour le choix des délégués du Québec, qui impliquait l'intervention des associations de comté de la Fédération libérale provinciale.

Ces tentatives d'imposer à partir du niveau provincial l'autorité de la Fédération sur l'ensemble des libéraux du Québec, fédéraux et provinciaux, ont 
échoué dans la pratique. Les règles furent facilement contournées, quelles qu'aient été les protestations de la Fédération.

Si on comprend l'objectif poursuivi par une telle démarche, eue n'en demeure pas moins ambiguë, dans la mesure où ces diverses ententes avaient pour effet d'institutionnaliser l'interpénétration des deux partis. Ainsi, au moment du départ de G.-É. Lapalme, ce problème des relations fédérales-provinciales à l'intérieur de la famille libérale n'était-il pas réglé. Le sentiment de certains était même qu'il était peut-être temps de mettre fin à ces tensions inutiles qui détournaient des vrais problèmes.

Ce qui n'apparaissait pas encore clairement à ce moment, c'est qu'une véritable orientation autonomiste du Québec ne pouvait qu'entrer en conflit avec le centralisme de l'État fédéral, surtout celui des libéraux, et que dès lors il deviendrait très difficile de maintenir une conception unitaire de l'ensemble de la famille libérale. Il faudrait développer un instrument de lutte et de réalisation entièrement voué à sa fonction provinciale.

Au cours de cette transformation, le Parti libéral du Québec - c'est le nom qu'il commence à prendre ou qui lui est attribué vers la fin des années 50 - en vient à préconiser toute une série de politiques, sociales, économiques, etc. qui ressortent d'une volonté de développer la plénitude des pouvoirs du Québec. Ces pratiques qui entraînent le développement du rôle de l'État poseront très tôt le problème du partage fiscal et celui des juridictions. Au moment du départ de G.-É. Lapalme, le Parti libéral est dans l'opposition à Ottawa et à Québec. Le retour au pouvoir à Québec en 1960, puis à Ottawa en 1963, allait modifier complètement la situation. Le Parti libéral du Québec avait mis en place des éléments nouveaux et les résultats de cette transformation devaient être marquants après 1960. C'est en effet à partir de cette date qu'une situation complètement nouvelle apparut et fit ressortir les rapports étroits qui existent entre le type de fédéralisme vécu et le type de système partisan qui lui convient.

Dans sa première phase de développement le fédéralisme canadien, fortement unitaire et centralisateur, s'est accompagné de partis politiques provinciaux relativement faibles et dépendants. Au moment où le fédéralisme canadien, vers la fin du XIXe siècle, se transforme et correspond davantage aux normes de cette forme d'État, le Parti libéral provincial est au pouvoir. Il n'éprouve pas de difficultés à préserver son autonomie, d'autant plus que le Parti libéral fédéral connaît des aléas électoraux très prononcés. Au moment où les libéraux fédéraux reviennent au pouvoir à partir de 1935 et entreprennent de poursuivre une politique beaucoup plus centralisatrice et interventionniste, aidés en cela par la guerre et inspirés par l'esprit de la Commission Rowen Sirois, les tensions entre libéraux fédéraux et provinciaux peuvent demeurer à un niveau acceptable. Le Parti libéral provincial, faible et démuni dans l'opposition, ne constituait pas 
véritablement une menace. La transformation opérée pendant cette période commence, cependant, nous l'avons vu, à poser problème.

L'arrivée au pouvoir d'un Parti libéral provincial, décidé à favoriser pleinement le développement de l'État québécois, en assumant l'héritage à la fois nationaliste et progressiste, introduisait au cœur même du fédéralisme centralisateur un élément conflictuel tout comme au cœur de la famille libérale. Au-delà des idéaux dits communs, les responsabilités réciproques des deux partis exigeaient une spécialisation encore plus poussée, une séparation. C'est ce que le congrès de 1964 a enregistré, c'est ce que les années qui ont suivi ont démontré. Pas plus sous Lesage que sous Bourassa, sous Ryan ou de nouveau sous Bourassa, et même au moment du référendum, une pratique libérale partisane analogue à celle qui a prévalu dans le passé ou même encore pendant la période de transition des années 50 n'a été possible. A l'origine de cette transformation fondamentale, on retrouve donc les effets à la fois voulus et insoupçonnés des changements survenus au sein du Parti libéral provincial des années cinquante.

\section{Références}

$\underline{\text { Retour à la table des matières }}$

Bergeron, G., Du Duplessisme au Johnsonisme. 1956-1966, Parti pris, Montréal, 1967.

Boily, R., "Les partis politiques québécois - perspectives historiques," dans V. Lemieux, Personnel et partis politiques au Québec, Boréal Express, Montréal, 1982, pp. 27-68.

Comeau, P.-A., "La transformation du Parti libéral québécois", dans V. Lemieux, Personnel et partis politiques au Québec, Boréal Express, Montréal, 1982, pp. 141-154 -,Le Bloc populaire. 1942-1948. Québec-Amérique, Montréal, 1982.

Lapalme, G.-É., Mémoires. I. Le Bruit des choses réveillées ; II Le Vent de l'oubli ; III. Le Paradis du pouvoir, Leméac, Montréal, 1969, 1971, 1973.

Nadeau, J.-M., Carnets politiques, Parti pris, Montréal, 1970. 
Thomson, D.C., Jean Lesage \& The Quiet Revolution, Macmillan of Canada, Toronto, 1984.

Fédération libérale provinciale du Québec, Procès-verbaux des congrès annuels, 1955, 1956, 1957, 1958, Procès-verbaux des réunions de l'Exécutif et du Conseil général, 1955 à 1959.

Le Devoir, La Presse, The Gazette, The Montreal Daily Star, Le Soleil, Couverture des congrès à la direction de 1950 et de 1958 et des congrès de la Fédération de 1955, 1956, 1957, 1958. 